Test Method

\title{
Characterizing the Constitutive Response and Energy Absorption of Rigid Polymeric Foams Subjected to Intermediate-Velocity Impact
}

\author{
Behrad Koohbor $^{\mathrm{a}}$, Addis Kidane ${ }^{1 \mathrm{a}}$, Wei-Yang Lu ${ }^{\mathrm{b}}$ \\ ${ }^{a}$ Department of Mechanical Engineering, University of South Carolina, 300 Main St, Columbia, SC 29208 \\ ${ }^{b}$ Sandia National Laboratories, Livermore, CA 94551-0969
}

\begin{abstract}
As an optimum energy-absorbing material system, polymeric foams are needed to dissipate the kinetic energy of an impact, while maintaining the impact force transferred to the protected object at a low level. Therefore, it is crucial to accurately characterize the load bearing and energy dissipation performance of foams at high strain rate loading conditions. There are certain challenges faced in the accurate measurement of the deformation response of foams due to their low mechanical impedance. In the present work, a non-parametric method is successfully implemented to enable the accurate assessment of the compressive constitutive response of rigid polymeric foams subjected to impact loading conditions. The method is based on stereovision high speed photography in conjunction with 3D digital image correlation, and allows for accurate evaluation of inertia stresses developed within the specimen during deformation time. Full-field distributions of stress, strain and strain rate are used to extract the local constitutive response of the material at any given location along the specimen axis. In addition, the effective energy absorbed by the material is calculated. Finally, results obtained from the proposed non-parametric analysis are compared with data obtained from conventional test procedures.
\end{abstract}

\footnotetext{
${ }^{1}$ Corresponding Author

Department of Mechanical Engineering (Room A132), University of South Carolina, 300 Main Street, Columbia, SC 29208 | Tel:

+1 (803) 7772502 | Fax:+1 (803) 7770106 | E-mail: kidanea@cec.sc.edu
} 
KEYWORDS: polymeric foam; direct impact; digital image correlation; inertia; energy absorption

\section{INTRODUCTION}

Owing to their superior energy absorption and acoustic characteristics, rigid polymeric foams are widely used in the aerospace and automotive industries. In addition to energy dissipation performance, advanced polymeric foams are also gaining attention in structural components, as well as the core material for strengthening hollow structures while maintaining the overall structural weight at low levels [1-3]. The enhanced energy dissipation properties of polymer foams are mainly due to their low mechanical impedance, whereas such low impedance is itself a direct result of the porous structure of these materials. Although the low impedance behavior makes polymeric foam the material of choice in applications where dynamic loading conditions are dominant, it makes characterization of the dynamic behavior of foams more challenging compared with solid non-porous structures. The main challenge is due to the relatively low elastic wave speed in these materials which results in a delayed state of stress equilibrium during loading [4]. There have been solutions proposed to compensate for the belated state of equilibrium in dynamic loading conditions, most of which are only applicable in studies utilizing split Hopkinson pressure bars (SHPB). Application of hollow and/or polymeric bars to reduce the impedance mismatch between bars and specimen [5-7], and pulse shaping techniques $[8,9]$ are the most common solutions proposed to tackle the challenges in dynamic testing of foams in SHPB.

There have been several studies focusing on experimental characterization of direct impact response of polymeric foams [10-12]. In direct impact loading, none of the above-mentioned solutions are applicable since the basis of the experimental approach is on the direct measurement 
of the applied impact force. A more general solution to compensate for the non-equilibrium stress condition in direct impact experiments is the use of short specimens [13]. The main benefit in using short specimens in direct impact studies is due to the significant reduction of the elastic wave reverberation time in order to achieve the quasi-static equilibrium in a shorter time. However, in the case of cellular materials, there exists a lower bound on the dimensions of the specimen subjected to impact testing due to the representative volume element (RVE) size required to maintain the continuum response of the material [14-16].

The challenges listed above call for a more general approach that (a) is independent of the specimen dimensions; (b) facilitates accurate assessment of the dynamic deformation response of the foam even where non-equilibrium stress conditions are present. Recent advances in high speed photography and full-field measurements have provided a path for more accurate study of the dynamic deformation of low impedance materials. In particular, recent advances in inverse identification of the constitutive response of materials have been established to be promising approaches providing accurate assessment of the high strain rate deformation response. The basic idea in the area of inverse identification from full-field measurement is the use of kinematics of the deformation (displacement and strain) to inversely identify the loads/stresses imposed on the object. The virtual fields method (VFM) is an example of such inverse analyses gaining extensive popularity in the experimental mechanics community [17].

For the specific case of impact loading of low impedance materials, direct identification of the constitutive response based on full-field measurements was proposed by Othman et al. [18], in which the contribution of inertia was also included in the analysis by computing the full-field acceleration developed in the impacted specimen. The same approach was implemented to study the direct impact response of various polymer foams at strain rates of up to $2500 \mathrm{~s}^{-1}[19,20]$. Our 
objective in the present work is to extend the previous idea by adopting the same non-parametric analysis to study the energy absorption characteristics of closed-cell polymeric foams subjected to intermediate-velocity direct impact. The term "intermediate-velocity" indicates that the impactor velocity is sufficiently high so that a static analysis may not be justified. In this range, the initial inertia forces are of considerable magnitude [21]. On the other hand, the impactor velocity is not high enough to significantly increase the influence of elastic wave and shock wave propagations in the material, therefore the term "intermediate-velocity" is adopted. The novel contribution in the present study is the consideration of dynamic deformation of the foam during loading and unloading in the analysis. Accordingly, impact experiments were designed in a way that a relatively large magnitude of plastic strain (> 20\%) is imposed on the specimens without causing total failure. Full-field displacement is used to determine the distribution of acceleration over the entire length of the specimen. Inertia stresses are computed from the acceleration, and then superimposed with the boundary-measured stress to determine the total axial stress magnitudes. A complete stress-strain hysteresis loop is acquired, enabling the study of energy dissipation characteristics of the utilized material.

\section{EXPERIMETNAL}

\subsection{Material and Specimen Geometry}

High density closed-cell polyurethane foam under the commercial name TufFoam35 was examined in this work [22]. Nominal bulk density of the as-received foam was measured inhouse by measuring the mass and volume, and found to be $560 \mathrm{~kg} / \mathrm{m}^{3}$ (35 pcf). Cellular structure of the material is presented in Fig. 1, exhibiting circular cells with a $150 \mu \mathrm{m}$ average diameter 
and $150 \mu \mathrm{m}$ cell-wall thickness. Elastic modulus of the foam at quasi-static conditions was measured in-house as $780 \mathrm{MPa}$. Cylindrical specimens of $28.8 \mathrm{~mm}$ in length and $25.3 \mathrm{~mm}$ in diameter were extracted from the as-received foam billet by the use of a hole saw. The application of a hole saw results in a smooth lateral surface finish with $\pm 0.1 \mathrm{~mm}$ dimensional accuracy. For image correlation purposes, a high contrast speckle pattern comprised of a black substrate with $80 \mu \mathrm{m}$ white particles was applied on the lateral surface of the specimen. Speckling was carried out using normal flat spray paints.

\subsection{Direct Impact Experiments}

Controlled direct impact was applied to the specimen using a shock tube apparatus. Details on the design aspects of the shock tube are beyond the scope of this work, but can be found elsewhere [19, 23]. A schematic representation of the utilized shock tube is depicted in Fig. 2. The speckled specimen was inserted on a custom fabricated fixture close to the muzzle of the shock tube. The custom fixture incorporates a piezoelectric load-cell, facilitating measurement of the reaction force at the rear side of the specimen. The load-cell used in this work was an 88.8 $\mathrm{kN}$ capacity PCB piezotronics ${ }^{\circledR}$ load-cell, designed primarily for impact force measurements. The specimen was placed in its position on the fixture with the use of lithium grease, which also serves as a lubricant to diminish the effect of friction.

To increase the momentum transfer to the specimen and achieve higher strain rates, a $0.07 \mathrm{~kg}$ aluminum projectile was placed inside the tube and shot directly at the foam. Velocity of the projectile at the tube exit can be manipulated by varying the number and/or thickness of the plastic diaphragms used to separate the driver and the driven sections of the shock tube (see Fig. 
2). In the present work, projectile velocity of $40.4 \mathrm{~m} / \mathrm{s}$ was achieved by using a plastic diaphragm with $0.1 \mathrm{~mm}$ total thickness. The impactor velocity was measured by tracking the aluminum projectile after release from the muzzle of the tube, just before in contact with the specimen. The velocity of the projectile is highly repeatable, with uncertainty level in this work of about $\pm 5 \%$, which corresponds to $\pm 2 \mathrm{~m} / \mathrm{s}$. More details on the projectile velocity measurement can be found in [20]. The projectile velocity regarded here falls well within the range of intermediate velocity impact for the case of the foam studied in this work. Impact experiments were designed in such a way that the material will deform up to a global strain at which surface cracks are visibly formed on the lateral surface of the specimen. Reproducibility of the results was confirmed by conducting at least three independent experiments following the same experimental procedure. Once the repeatability was confirmed, typical results were extracted and presented in this work.

\subsection{High Speed Imaging and Digital Image Correlation}

High speed stereovision imaging in conjunction with digital image correlation was used in order to analyze and quantify the deformation of the impacted specimens. The imaging setup was composed of a pair of high speed Photron SA-X2 cameras each equipped with a $100 \mathrm{~mm}$ macro lens. The cameras were mounted vertically in a custom made fixture, acquiring images from the lateral surface of the impacted foam specimen, as seen in Fig. 3. Cameras were triggered by the use of the signal sent from the oscilloscope attached to the load-cell. Connection of the cameras to the oscilloscope in this work facilitates synchronization of the load-cell data collection with the image acquisition rate. Stereo images were collected at a rate of 150,000 frames per second at full-field resolution of $256 \times 216$ pixel $^{2}$. Images acquired during deformation stage were 
processed and analyzed in the image correlation software Vic-3D (Correlated Solutions, Inc.). Details of the image correlation process are listed in Table 1.

The default Cartesian coordinate system of the measurements in Vic-3D was switched to the cylindrical coordinate systems using the built-in coordinate tool in the software Vic-3D.

Distribution of the full-field displacement and strain was computed in this software. Temporal derivatives were computed from full-field displacement and strains using simple central difference scheme as:

$\dot{\varepsilon}_{i j}(t)=\frac{\varepsilon_{i j}(t+\Delta t)-\varepsilon_{i j}(t-\Delta t)}{2 \Delta t}$

$v_{i}(t)=\frac{u_{i}(t+\Delta t)-u_{i}(t-\Delta t)}{2 \Delta t}$

$a_{i}(t)=\frac{u_{i}(t+\Delta t)-2 u_{i}(t)+u_{i}(t-\Delta t)}{\Delta t^{2}}$

where $u_{i}$ and $\varepsilon_{i j}$ denote the components of the displacement and strain, respectively.

Parameters $\dot{\varepsilon}_{i j}, v_{i}$ and $a_{i}$ represent strain rate, velocity and acceleration, respectively. $\Delta t$ is the inter-frame time $(=6.67 \mu \mathrm{s})$.

\section{DATA ANALYSIS}

The objective in this work is to take the effect of inertia loading during dynamic deformation of the foam specimen into account. This goal is achieved through the application of the nonparametric analysis introduced in [18-20]. In this approach, the inertia stress is calculated from 
the full-field data obtained from DIC. The first step in this approach is to consider the general dynamic stress equilibrium, expressed as:

$\sigma(z, t)=\sigma^{\text {boundary }}+\sigma^{\text {inertia }}$

where:

$\sigma^{\text {boundary }}=\sigma(0, t) \quad$ and $\quad \sigma^{\text {inertia }}=\int_{\xi=0}^{\xi=z} \rho(\xi, t) a(\xi, t) d \xi$

Eq. 4 is a direct solution of the equation of stress equilibrium in the absence of body forces and shear stresses, and states that the value of the axial stress, $\sigma$, at location $z$ and time $t$ can be evaluated using the stress measured at specimen end, i.e. $z=0$, plus the inertia stress [18-20]. The inertia stress is expressed by the integral term in Eq. 5 as a function of the spatial variation of density, $\rho$, and axial acceleration, $a$. This inertia stress can be evaluated from the full-field data. Using the full-field distribution of acceleration and density, the integral term in Eq. 5 can be approximated by a simple summation as:

$$
\int_{z=0}^{z=L} \rho(z, t) a_{z}(z, t) d z \approx \sum_{i=1}^{n} \rho_{(i)} a_{(i)} S_{(i)}
$$

where, $\rho_{(i)}$ and $a_{(i)}$ represent the average values of density and axial acceleration in section $i$. The term $S_{(i)}$ denotes the thickness of section $i$, as illustrated in Fig. 4. Variation of material density in time and space is included in this approach by using a recently proposed model [20] that enables the calculation of local instantaneous density as a function of initial density, $\rho_{0}$, local Poisson's ratio, $v(z, t)$, and local axial strain value, $\varepsilon(z, t)$, expressed as: 
$\rho(z, t)=\rho_{0}[\exp (\varepsilon(z, t))]^{2 v(z, t)-1}$

Having obtained the variations of axial acceleration and material density in time and space, inertia stress at any desired location along the length and at any given time can be evaluated using Eq. 6. Superimposing this inertia stress with the boundary measured stress, local axial stress along the specimen length can be determined from Eq. 4. The stress values calculated from Eq. 4 are synchronized with the axial strain values, as the load data collection and image acquisition are initiated at the same instant and carried out at the same rate; therefore, local stress-strain curves at any given position along specimen length can be extracted.

Specific energy (energy per unit volume) can be determined from the area below the global stress-strain curve. To evaluate the specific energy consumed by the foam specimen, average stress-strain curves are considered first. Spatially averaged stress and strain values are calculated as:

$\overline{\sigma(t)}=\frac{\int_{0}^{L(t)} \sigma(z, t) d z}{L(t)}$

$\overline{\varepsilon(t)}=\frac{\int_{0}^{L(t)} \varepsilon(z, t) d z}{L(t)}$

where $\overline{\sigma(t)}$ and $\overline{\varepsilon(t)}$ are the spatially-averaged axial stress and axial strain, respectively. In the case of the application of full-field data, spatial averaging can be carried out using simple numerical integration. Having obtained synchronized values for $\overline{\sigma(t)}$ and $\overline{\varepsilon(t)}$, average stressstrain curve of the specimen can be generated by plotting $\bar{\sigma}$ versus $\bar{\varepsilon}$. Finally, the specific energy of the specimen, $W$, can be determined as: 


$$
W=\int_{0}^{\bar{\varepsilon}} \bar{\sigma} d \bar{\varepsilon}
$$

\section{RESUTLS AND DISCUSSION}

\subsection{Nominal Stress-Strain Curve}

Fig. 5 shows 20 gray level images recorded during loading and unloading of the specimen. Physical dimensions of each individual grey-scale image in Fig. 5 are approximately $33 \times 28$ $\mathrm{mm}^{2}$, covering the entire length of the specimen. The reference time $t=0 \mu \mathrm{s}$ indicates the instant at which the contact between specimen and the projectile is established. It is shown that the specimen is loaded in compression during the first $400 \mu \mathrm{s}$ after the impact. Unloading of the specimen occurs after $t \approx 400 \mu \mathrm{s}$, as clearly indicated in Fig. 5 by the gradual movement of the projectile to the right side.

Variation of specimen length with time was calculated from the displacement of the impacted side. Fig. 6 depicts the variation of specimen length, along with the boundary-measured reaction force. Note that the reaction force is compressive, but is plotted in Fig. 6 as a positive quantity for convenience. Also for convenience, the term "reaction force" is replaced by the "boundarymeasured force" hereafter. Variation of the specimen length shows a progressive decrease in the first 65 images recorded after the impact, reaching a minimum at about $t \approx 430 . \pm 3 \mu \mathrm{s}$. On unloading, the specimen length starts to increase again to reach a final value of $26.6 \mathrm{~mm}$ after complete unloading. The boundary-measured force also indicates a sharp rise in the first $30 \mu \mathrm{s}$ after the impact, and remains relatively constant for a duration of $\sim 420 \mu \mathrm{s}$, followed by a gradual decrease during the unloading stage. Using the boundary measured force and displacement, 
engineering (nominal) stress-strain curve was extracted and is plotted in Fig. 7. The engineering stress here is simply determined by dividing the boundary-measured force by the original cross sectional area. Engineering strain was calculated as the ratio of the change in specimen length $(\Delta L)$ and the original length $\left(L_{0}\right)$. Note that the engineering stress-strain curve here is regarded as the conventional approach, since the calculation of true stress requires the determination of instantaneous cross sectional area, a concept that may not be accurately achieved for compressible closed-cell foams with considerable radial expansion. In this regard, in the case of compressible cellular solids such the one studied in this work, volume constancy is not a valid assumption and, therefore, the true stress values cannot be directly calculated using the correlation between the instantaneous length and cross sectional area.

The nominal stress-strain curve in Fig. 7 indicates a noticeable plateau at the beginning of the curve. The small plateau seen here is attributed to the delayed state of stress equilibrium at the early stages of deformation [19]. In this transient duration, there has not been sufficient time for the stress wave to traverse the entire length of the specimen and reach the load-cell side; however, the deformation has already initiated from the impacted side. Consequently, deformation occurs in the specimen before the force can be recorded by the load-cell. Except the small plateau observed at early deformation time, engineering stress-strain curve plotted for the impacted specimen is typical of the constitutive response documented for closed-cell foams under compression [11]. The curve exhibits three distinct regions. At small nominal strains (< $3 \%$ ) the material exhibits linear elastic response. Gradual collapse of the cell structure at larger nominal strains progresses at relatively constant nominal stress, indicating a stress plateau over an engineering strain range of $6 \%$ to $15 \%$. During the unloading phase, the stress decreases nonlinearly with the strain, resulting in a progressively decreased slope. Finally, a residual 
permanent nominal strain of $\sim 9 \%$ is retained in the specimen after complete unloading. The lower apparent slope during unloading is attributed to cellular scale damage and the formation of cracks in the specimen. Fig. 8 illustrates the photograph of the vertically-sectioned deformed specimen, evidencing the formation of small visible cracks inside the impacted specimen. Note that the material examined in this work does not exhibit any significant densification stage in its nominal stress-strain curve.

\subsection{Deformation Analysis Based on Full-Field Measurements}

Full-field distribution of axial displacement during the loading is shown in Fig. 9. The area over which full-field results are shown covers more than $85 \%$ of the entire domain of interest (speckle pattern). Contour maps in Fig. 9 show relatively uniform axial displacement distribution in the hoop direction, indicating insignificant shear deformation during the impact tests in this work. Accordingly, the analysis proceeded based on the formulations proposed for the uniaxial stress state.

Distribution of axial strain over the entire area of interest is shown in Fig. 10. Strain maps in Fig. 10 exhibit the formation of relatively uniform axial strain value in the hoop direction. On the other hand, the axial strain varies along the specimen length, clearly showing high strain region formed over the middle sections of the specimen. The presence of high strain domains in the middle length of the specimen is due to boundary effects. Friction at specimen ends can partially restrain the lateral expansion of the specimen in the vicinity of the two ends, resulting in larger deformation concentration at middle sections. This statement is supported by observing radial 
strain maps in Fig. 11, where larger radial strains are shown to have developed in the middle length of the specimen.

Axial strain rate applied on the specimen was determined by differentiating the spatiallyaveraged axial strain values with respect to time. Fig. 12 shows the variation of axial strain rate with time. A maximum average compressive strain rate of $1000 \mathrm{~s}^{-1}$ was applied to the specimen at the time of peak load. The strain rate curve then shows a gradual decrease and changes sign at $t=400 \mu$ s. This sign change is due to the reversion of acceleration and is discussed in detail later in this section. The strain rate remains zero after $t=1100 \mu$ s, i.e. the instant of complete unloading. Note that the error bars in Fig. 12 indicate the standard deviation of the entire population of strain rate data within the area of interest. This standard deviation here may be used as measure for the spatial variability of axial strain rate, confirming a maximum $150 \mathrm{~s}^{-1}$ spatial variation in the axial strain rate values along the specimen axis during the loading stage, i.e. $0<t<400 \mu$ s.

Using the local evolutions of axial and radial strains, the apparent Poisson's ratios at various locations along specimen axis was estimated following the procedure detailed in [19]. Fig. 13 shows the variation of local radial strain with axial strain extracted from five representative sections along the specimen axis. It is clearly seen that the Poisson's effect varies considerably along specimen length, indicating different apparent Poisson's ratios. Poisson's ratios extracted from different sections are detailed in Table 2. As expected, higher Poisson's ratios are obtained at sections located mostly on the middle length of the specimen. On the other hand, the Poisson's ratio values shown in Table 2 are all less than 0.5. This confirms that the material undergoes partial bulk compression resulting in an increase in density. This compressible response observed here makes the conventional calculation of true stress-strain response of the material challenging. 
The challenge here will be due to the impractical use of the concept of volume constancy in establishment of a correlation between the specimen length and its cross sectional area. This was discussed in more details earlier in Sec. 4.1.

Variation of density with time was determined using Eq. 7. Fig. 14 depicts the variation of local density at various locations along the specimen axis. All curves indicate an initial increase associated with the loading stage, followed by a drop as the result of unloading and deformation recovery. The density curves reach a plateau after $t \approx 1100 \mu \mathrm{s}$. Maximum local change of density occurs on $A_{1}$ and $A_{5}$, i.e. the representative sections located near to specimen ends. This is due to the relatively smaller lateral expansions that occurred within areas more closely associated with the specimen boundary, on which lower Poisson's ratios were also observed (see Table 2). On the other hand, a relatively smaller change of density is indicated on sections $\mathrm{A}_{3}$ and $\mathrm{A}_{4}$, where higher radial expansions were determined (see Fig. 11). The average density as a function of time was determined for the material, showing a maximum of $\sim 5 \%$ increase in density at the peak load. This average density change was later used along with Eq. 6 to facilitate the calculation of inertia effects based on boundary measured data.

Curves showing local evolution of axial acceleration are plotted in Fig. 15. All local curves show a sudden drop during the first $30 \mu$ s after the impact. This is followed by a sharp deceleration and a sign change occurred at $t=50 \mu \mathrm{s}$. The oscillations observed in the axial acceleration curves is attributed to the elastic wave reverberation along specimen axis. Based on the elastic modulus of the material measured at quasi-static conditions $(E=780 \mathrm{MPa})$, the time required for the stress equilibration was determined as $73.2 \mu \mathrm{s}$. This characteristic time is equivalent to the time required for three consecutive reverberations of the elastic wave in the material [24], which is also found to be equal to the time of sharp variations in the acceleration curves during the first 
$\sim 75 \mu \mathrm{s}$ after the impact. Acceleration curves indicate slight change in value after the transient duration (i.e. after $t \approx 75 \mu \mathrm{s}$ ). Further oscillations seen in the acceleration curves are basically due to numerical noise sourced from the temporal noise in the displacement data.

The significant variations observed in the local acceleration response of the material result in the development of substantial inertia stresses in the specimen. This inertia stress was calculated from the spatial length-wise variation of acceleration and density, as discussed earlier in Eq. 5. Fig. 16 shows the variation of inertia stress along the specimen length calculated at different times after the impact. The magnitude of the inertia stress is shown to be highest at the impacted side of the specimen at all times. This observation is consistent with the acceleration curves shown earlier in Fig. 15, in which the highest accelerations were found to develop over the regions more closely associated with the impacted side of the specimen. Another point worth noting is that the inertia stresses developed in the specimen remain compressive (negative) during the first $50 \mu \mathrm{s}$ after the impact. During this time, the compressive inertia stresses add up to the compressive boundary-measured stress and increase the overall axial stress magnitude applied on the specimen at any given location along its axis. Afterwards, on reversion of the axial acceleration, inertia stresses will become tensile (positive) and tend to decrease the overall stresses in the specimen by cancelling out some portion of the compressive stress measured at the specimen end.

Superimposing the obtained inertia stress with the true stress measured at the specimen boundary, local evolution of total axial stress can be evaluated. Fig. 17 shows the variation of total axial stress with time. Note that, for convenience, the compressive stresses are plotted in positive values hereafter. Substantial length-wise variability in total stress is observed during the initial $100 \mu \mathrm{s}$ time after the impact. The degree of such spatial variability tends to reduce at longer durations, as 
the damping of the acceleration occurs and the state of quasi-static stress equilibrium is achieved. Similarly, local length-wise variation of strain can be readily extracted as a direct output of the image correlation analysis, finally facilitating the extraction of local stress-strain curves at different locations along the specimen length. The local stress-strain curves can be averaged using Eqs. 8 and 9 to give an effective average constitutive response for the material. Fig. 18 illustrates the effective stress-strain curve obtained by averaging all the local curves extracted along the specimen axis. Comparing Fig. 18 with the nominal engineering stress-strain curve shown earlier in Fig. 7, there is noticeable difference between the two curves at the early stages of deformation. In particular, the delayed stress rise time is no longer present in the curve obtained from the full-field approach. This is indeed expected since the influence of inertia and the non-equilibrium state of stress are already accounted for in the non-parametric approach. Finally, the stress-strain curve shown in Fig. 18 gives a more realistic measure for the actual constitutive response of the examined material. This can be further confirmed by extracting the slope of the curve at the elastic deformation regime. The elastic modulus determined from the curve presented in Fig. 18 is 810 $\mathrm{MPa}$, the value of which is slightly higher than the elastic modulus of the material measured at quasi-static loading conditions, i.e. $780 \mathrm{MPa}$. The increased elastic modulus measured from data presented in Fig. 18 can be justified through the visco-elastic nature of the material employed here. Note that, conventionally, due to transient state of deformation at the early stages of the dynamic loading, measuring elastic modulus at high strain rate is impossible. For example, applying the same procedure to the nominal stress-strain curve shown in Fig. 7 results in an elastic modulus of $<200 \mathrm{MPa}$, which is significantly lower than the quasi-static modulus of the material, and thus may not be regarded as a reliable value. However, in the current work, the dynamic equilibrium was considered from the beginning of the loading by accounting for the inertia stress, which makes 
the analysis significantly more reliable. Another significant difference in the stress-strain curves obtained using conventional and full-field approaches is that the conventional methodology assumes uniform strain distribution along gauge area and, therefore, does not take the influence of any local deformation into account. On the other hand, the stress-strain analysis based on the fullfield measurement does include the effects due to local deformation. The experimental observation depicted in Fig. 10 clearly confirms that the strain is not uniform across the length of the specimen.

Finally, the specific energy absorbed by the material calculated based on the two methods, i.e. conventional and the full-field stress analysis, are compared in Fig. 19. For this purpose, the area below stress-strain curve was evaluated as the specific energy magnitude absorbed during a full loading-unloading cycle. As shown in Fig. 19, the value of specific energy calculated using the full-field data is significantly higher compared with the value calculated following conventional method which is $20 \%$ lower. This is again due to the fact that, by accounting the local deformation, compressibility and inertia, the full field method helps to determine more realistic constitutive response of the material.

\section{CONCLUSIONS}

Constitutive and energy performance response of closed-cell polymeric foams subjected to intermediate-velocity impact were studied. Controlled direct impact was applied to foam specimens and the stress-strain response was investigated by two different methods. Nominal stress-strain response was first evaluated using simple definitions of engineering stress and engineering strain. It was shown that the nominal stress-strain response is incapable of predicting the accurate constitutive response of the foam, due to limitations associated with transient stress 
state, material compressibility and local length-wise variation of axial and radial strains. The deformation analysis of the impacted foam was also conducted using full-field measurements facilitated by the use of high speed photography and 3D digital image correlation. The analysis performed based on the full-field deformation measurements was shown to be able to accurately predict the deformation response of the material accounting for inertia loading, material compressibility and the spatial variation of strain. Specific energy absorbed during a complete loading-unloading cycle was determined using the two methods. It was shown that the application of conventional methodology, i.e. considering nominal stress-strain response, can underestimate the energy absorption characteristics of the polymeric foam specimen by $20 \%$.

\section{ACKNOWLEDGEMENTS}

Sandia National Laboratories is a multi-program laboratory managed and operated by Sandia Corporation, a wholly owned subsidiary of Lockheed Martin Corporation, for the U.S. Department of Energy's National Nuclear Security Administration under contract DE-AC0494AL85000. 


\section{REFERENCES}

[1] L.J Gibson, M.F Ashby. Cellular solids: Structure and properties. 2nd ed. Oxford. 1997

[2] N. Gardner, E. Wang, P. Kumar, A. Shukla. Blast mitigation in a sandwich composite using graded core and polyurea interlayer. Exp Mech 2012; 52: 119.

[3] B. Koohbor, A. Kidane. Design optimization of continuously and discretely graded foam materials for efficient energy absorption. Mater Design 2016; 102: 151.

[4] H. Zhao. Testing of polymeric foams at high and medium strain rates. Polym Test 1997; 16: 507.

[5] A.S Yunoshev, V.V Sil'vestrov. Development of the polymeric split Hopkinson bar technique. J Appl Mech Tech Phys 2001; 42(3): 558.

[6] W. Chen, B. Zhang, M.J Forrestal. A split Hopkinson bar technique for low-impedance materials. Exp Mech 1999; 39(2): 81.

[7] A. Kidane. On the Failure and Fracture of Polymer Foam Containing Discontinuities. ISRN Mater Sci 2013, DOI:10.1155/2013/408596.

[8] W. Chen, F. Lu, N. Winfree. High strain rate compressive behavior of a rigid polyurethane foam with various densities. Exp Mech 2002; 42(1): 65.

[9] B. Song, W. Chen, D.J Frew. Dynamic compressive response and failure behavior of an epoxy syntactic foam. J Compos Mater 2004; 38(11): 915.

[10] J. Zhang, N. Kikuchi, V. Li, A. Yee, G. Nusholtz. Constitutive modeling of polymeric foam material subjected to dynamic crash loading. Int J Impact Eng 1998; 21(5): 369. 
[11] M. Avalle, G. Belingardi, R. Montanini. Characterization of polymeric structural foams under compressive impact loading by means of energy-absorption diagram. Int J Impact Eng 2001; 25: 455.

[12] S. Ouellet, S. Cronin, M. Worswick. Compressive response of polymeric foams under quasistatic, medium and high strain rate conditions. Polym Test 2006; 25: 731.

[13] S. Ouellet, D.S Cronin, J. Moulton, E.O Petel. High rate characterization of polymeric closed-cell foams: Challenges related to size effects. In: Chalivendra V, Song B, Casem D (Editors), Dynamic behavior of materials - Volume 1, Proceedings of the 2012 Annual Conference on Experimental and Applied Mechanics, Springer, 2013, pp 21-28. DOI 10.1007/978-1-4614-4238-7_4

[14] S. Kanaun, O. Tkachenko. Representative volume element and effective elastic properties of open cell foam materials with random microstructures. J Mech of Mater Struct 2007; 2(7): 1607.

[15] J. Alsayednoor, P. Harrison, Z. Guo. Large strain compressive response of 2-D periodic representative volume element for random foam microstructures. Mech Mater 2013; 66: 7.

[16] C. Tekoglu, L.J Gibson, T. Pardoen, P.R Onck. Size effects in foams: Experiments and modeling. Prog Mater Sci 2011; 56: 109.

[17] F. Pierron, M. Grédiac. The virtual fields method: Extracting constitutive mechanical parameters from full-field deformation measurements. Springer, NY, 2012.

[18] R. Othman, S. Aloui, A. Poitou. Identification of non-homogeneous stress fields in dynamic experiments with a non-parametric method. Polym Test 2010; 29: 616. 
[19] B. Koohbor, S. Mallon, A. Kidane, W.Y Lu. The deformation and failure response of closed-cell PMDI foams subjected to dynamic impact loading. Polym Test 2015; 44: 112.

[20] B. Koohbor, A. Kidane, W.Y Lu, M.A Sutton. Investigation of the dynamic stress-strain response of compressible polymeric foam using a non-parametric analysis. Int J Impact Eng 2016; 91: 170.

[21] P.O Sjoblom, J.T Hatness. On low-velocity impact testing of composite materials. J Compos Mater 1988; 22: 30 .

[22] W.Y Lu. Mechanical characterization of rigid polyurethane foams. Sandia Report 2014: SADN2014-20708.

[23] S. Mallon, B. Koohbor, A. Kidane, M.A Sutton. Fracture behavior of prestressed composites subjected to shock loading: A DIC-based study. Exp Mech 2015; 55: 211.

[24] E.D.H Davies, S.C Hunter. The dynamic compression testing of solids by the method of the split Hopkinson pressure bar. J Mech Phys Solid 1963; 11(3): 155. 


\section{Figure Captions}

Fig. 1- Cellular structure of the examined foam.

Fig. 2- Schematic of the shock tube in its entirety, with a magnified view of the load fixture depicted in (b).

Fig. 3- Experimental setup showing the shock tube and the stereovision camera system.

Fig. 4- Cylindrical specimen sliced into a finite number of thinner sections, used to calculate the inertia stress applied at each point along the specimen axis.

Fig. 5- Gray level images. $\mathrm{t}=0 \mu$ s indicates the moment of impact.

Fig. 6- Variation of boundary-measured force and specimen length in time.

Fig. 7- Engineering (nominal) stress-strain curve during loading and unloading, calculated from boundary-measured force and specimen length.

Fig. 8- Photograph showing the vertical section of the specimen after deformation. Arrows mark visible cracks.

Fig. 9- Contour maps showing the full-field distribution of axial displacement at selected times after the impact. $\mathrm{t}=0 \mu \mathrm{s}$ indicates the instant of impact.

Fig. 10- Contour maps showing the full-field distribution of axial strain at selected times after the impact.

Fig. 11- Contour maps showing the full-field distribution of radial strain at selected times after the impact. 
Fig. 12- Variation of strain rate in time. Error bars indicate the spatial variability of strain rate.

Fig. 13- (a) Variation of local radial strain with axial strain extracted from five representative sections shown in (b).

Fig. 14- Variation of local density in time for the representative sections shown in Fig. $13 b$.

Fig. 15- Variation of local axial acceleration with time, extracted from the representative locations shown in Fig. 13b.

Fig. 16- Variation of inertia stress with respect to the normalized length of the specimen at different instants after impact, determined using full-field data.

Fig. 17- Variation of total (inertia plus boundary measured) stress with time at different locations in specimen length.

Fig. 18- True stress-strain curve during loading and unloading, calculated from full-field data.

Fig. 19- Comparing specific energy values determined from two different methods. 


\section{TABLES}

Table 1- Image acquisition and correlation parameters used in the present study

\begin{tabular}{|ll|}
\hline Camera system & Photron SA-X2 $(\times 2)$ \\
Frame rate & $150,000 \mathrm{fps}$ \\
Interframe time & $6.67 \mu \mathrm{s}$ \\
Exposure time & $293 \mathrm{~ns}$ \\
Image resolution & $256 \times 216 \mathrm{pixel}^{2}$ \\
Pixel-to-length ratio & $7.75 \mathrm{pixel}^{\mathrm{mm}}$ \\
\hline Full-field measurement technique & $3 \mathrm{D} \mathrm{DIC}(\mathrm{Vic}-3 \mathrm{D})$ \\
Subset & $21 \times 21 \mathrm{pixel}^{2}$ \\
Step & 1 pixel \\
Strain filtering (filter size) & Gaussian $(15)$ \\
Temporal smoothing & None \\
Matching criterion & Normalized squared differences \\
Interpolation & Optimized 8 -tap interpolation \\
Shape function & Affine \\
Strain resolution & $480 \mu \varepsilon$ \\
Acceleration resolution & $2 \times 10^{3} \mathrm{~m} . \mathrm{s}^{-2}$ \\
\hline
\end{tabular}

Table 2- Poisson's ratios extracted from different sections shown in Fig. 13b

\begin{tabular}{|c|c|c|c|c|c|c|}
\hline Section & $\mathrm{A}_{1}$ & $\mathrm{~A}_{2}$ & $\mathrm{~A}_{3}$ & $\mathrm{~A}_{4}$ & $\mathrm{~A}_{5}$ & Average \\
\hline Poisson's ratio & 0.358 & 0.382 & 0.426 & 0.407 & 0.329 & 0.386 \\
\hline
\end{tabular}




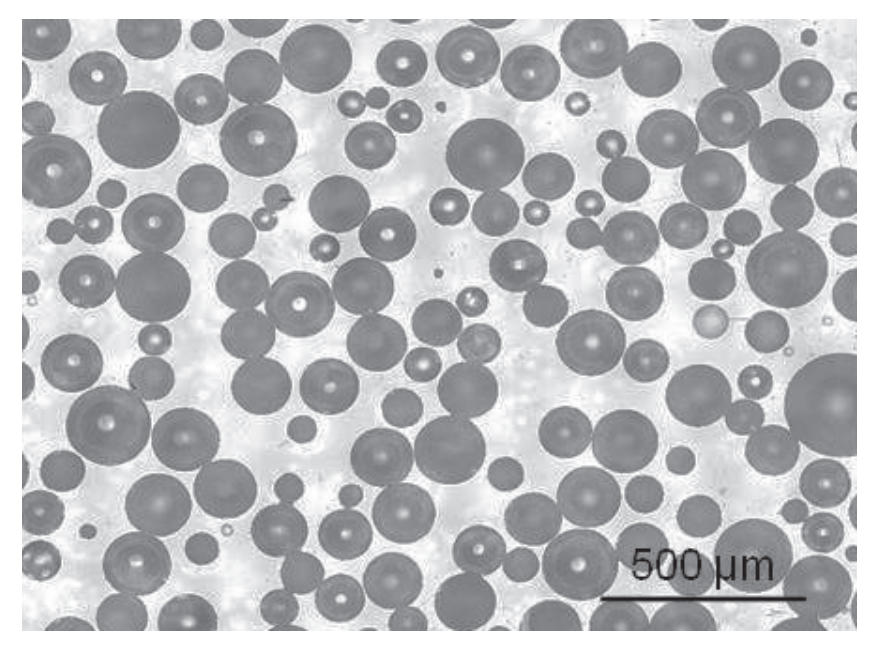

Fig. 1- Cellular structure of the examined foam.

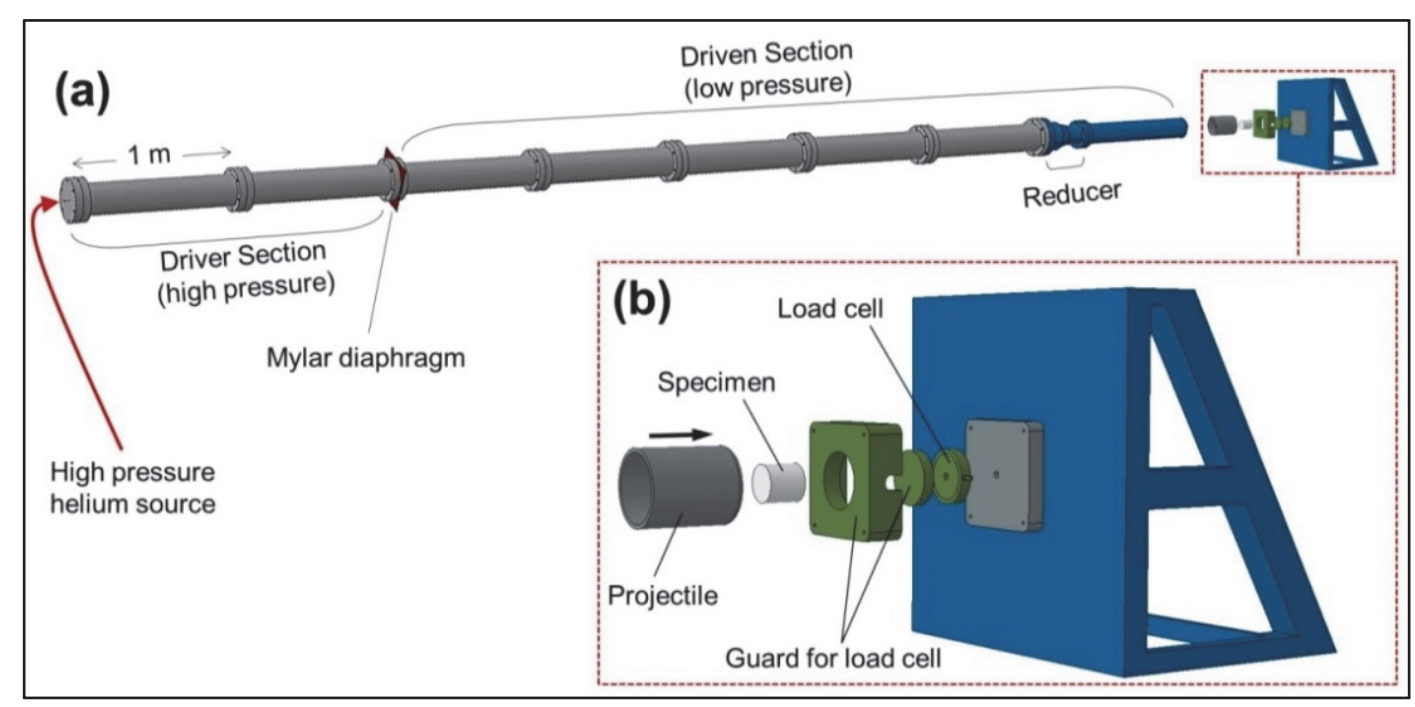

Fig. 2- Schematic of the shock tube in its entirety, with a magnified view of the load fixture depicted in (b). 


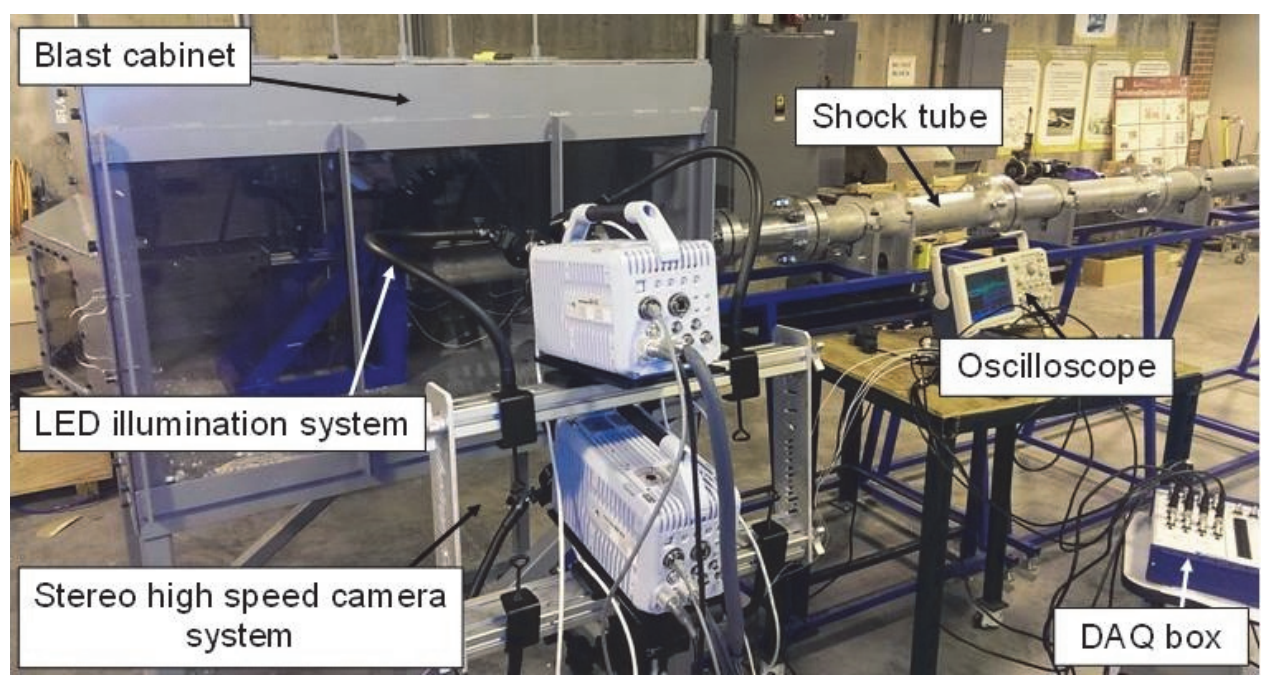

Fig. 3- Experimental setup showing the shock tube and the stereovision camera system.

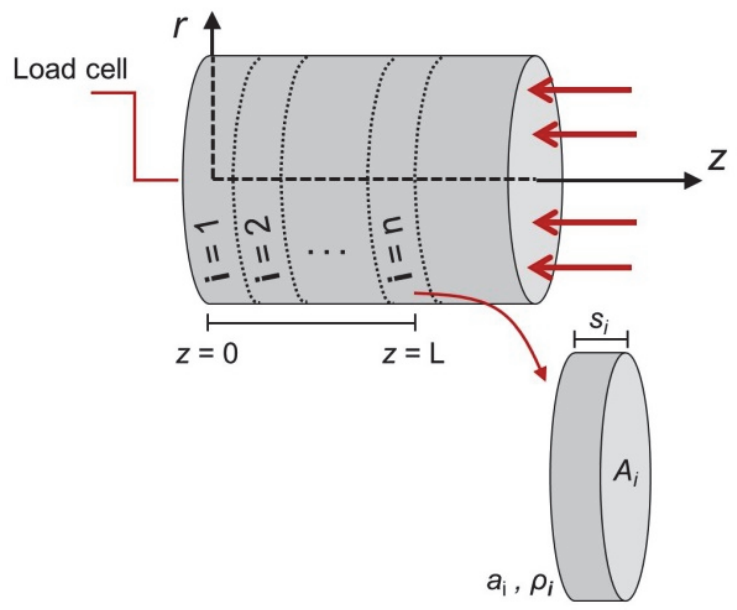

Fig. 4- Cylindrical specimen sliced into a finite number of thinner sections, used to calculate the inertia stress applied at each point along the specimen axis. 

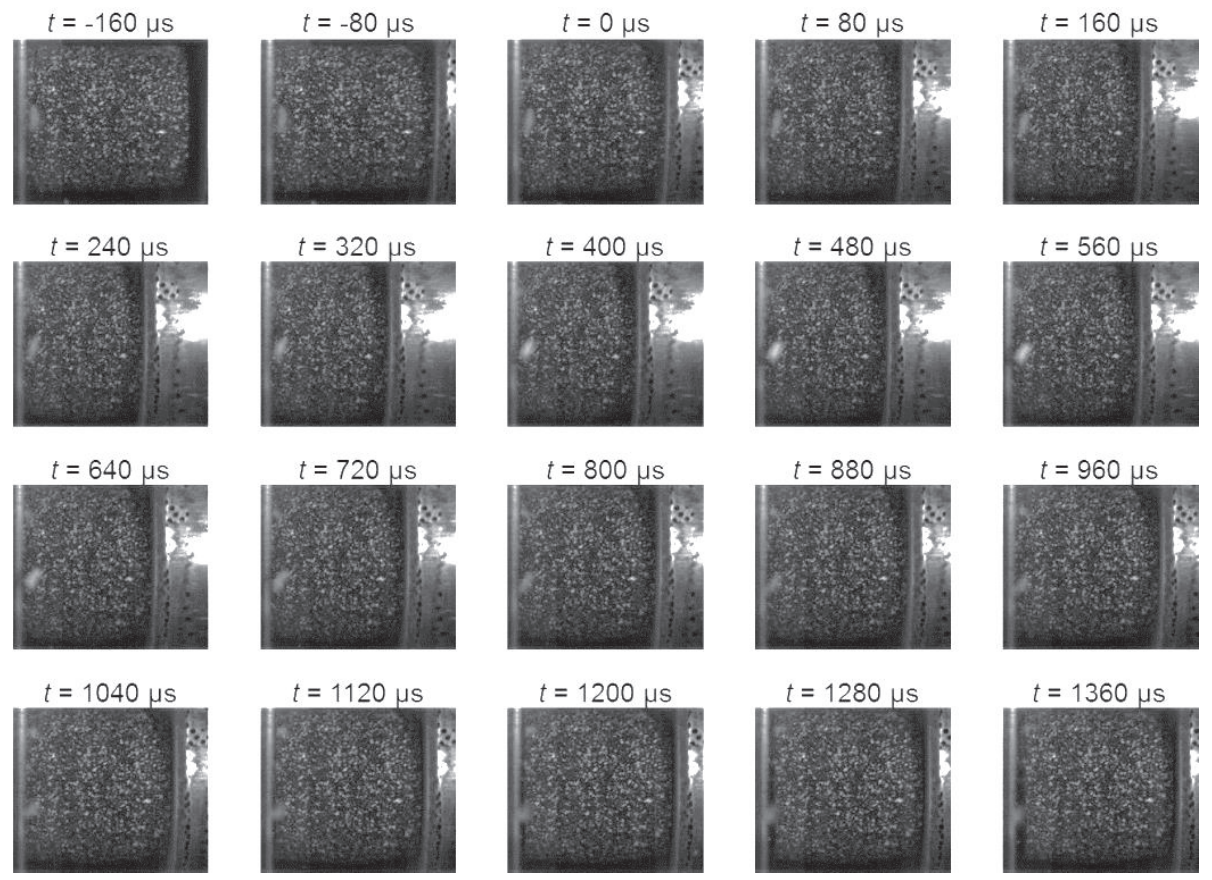

Fig. 5- Gray level images. $t=0 \mu$ s indicates the moment of impact.

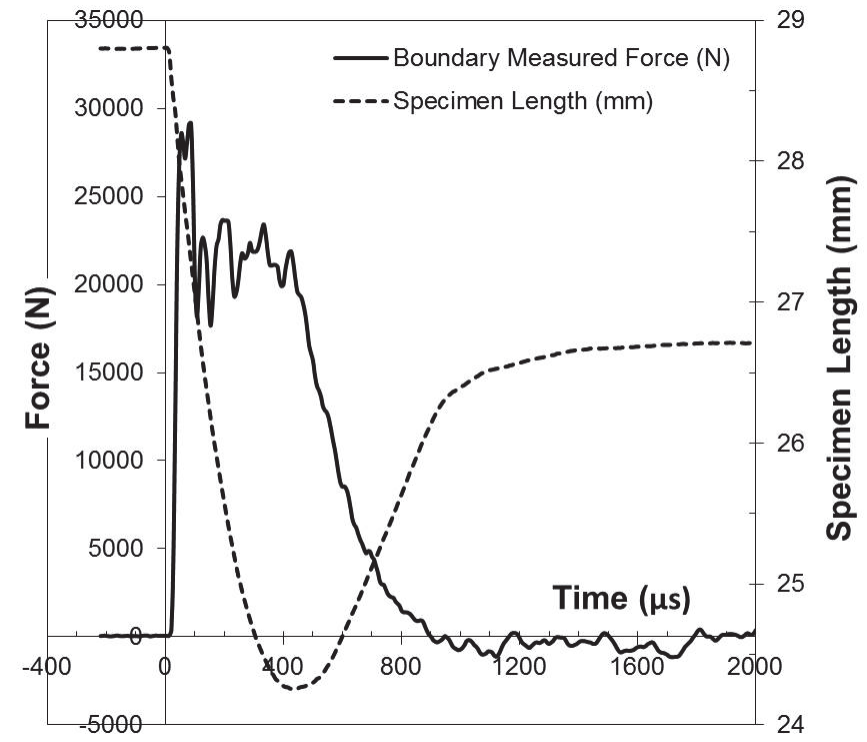

Fig. 6- Variation of boundary-measured force and specimen length in time. 


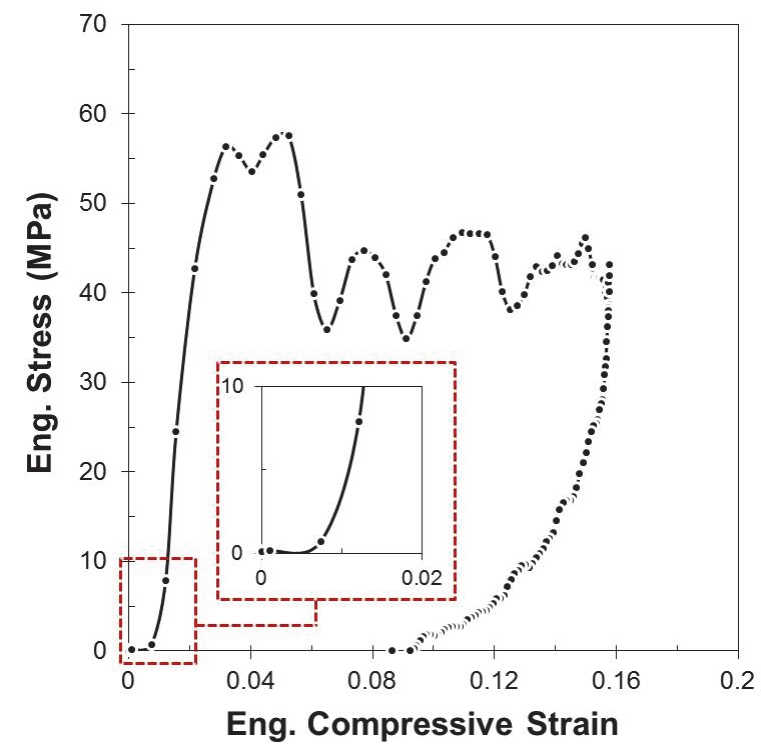

Fig. 7- Engineering (nominal) stress-strain curve during loading and unloading, calculated from boundary-measured force and specimen length.

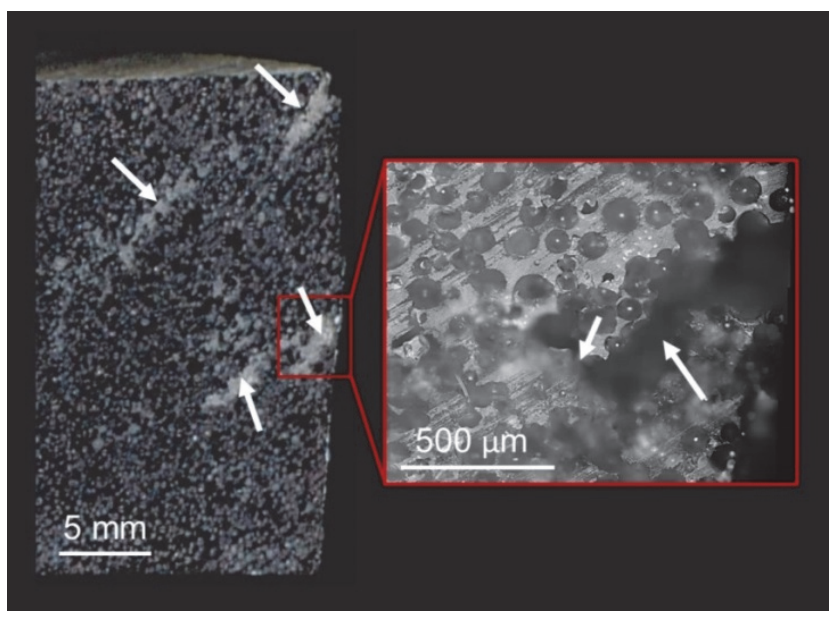

Fig. 8- Photograph showing the vertical section of the specimen after deformation. Arrows mark visible cracks. 


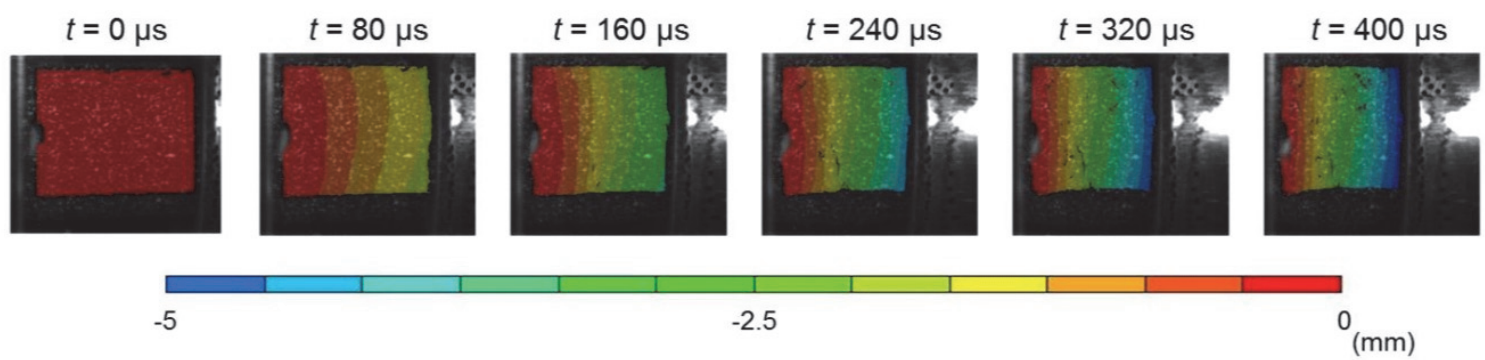

Fig. 9- Contour maps showing the full-field distribution of axial displacement at selected times after the impact. $t=0 \mu \mathrm{s}$ indicates the instant of impact.
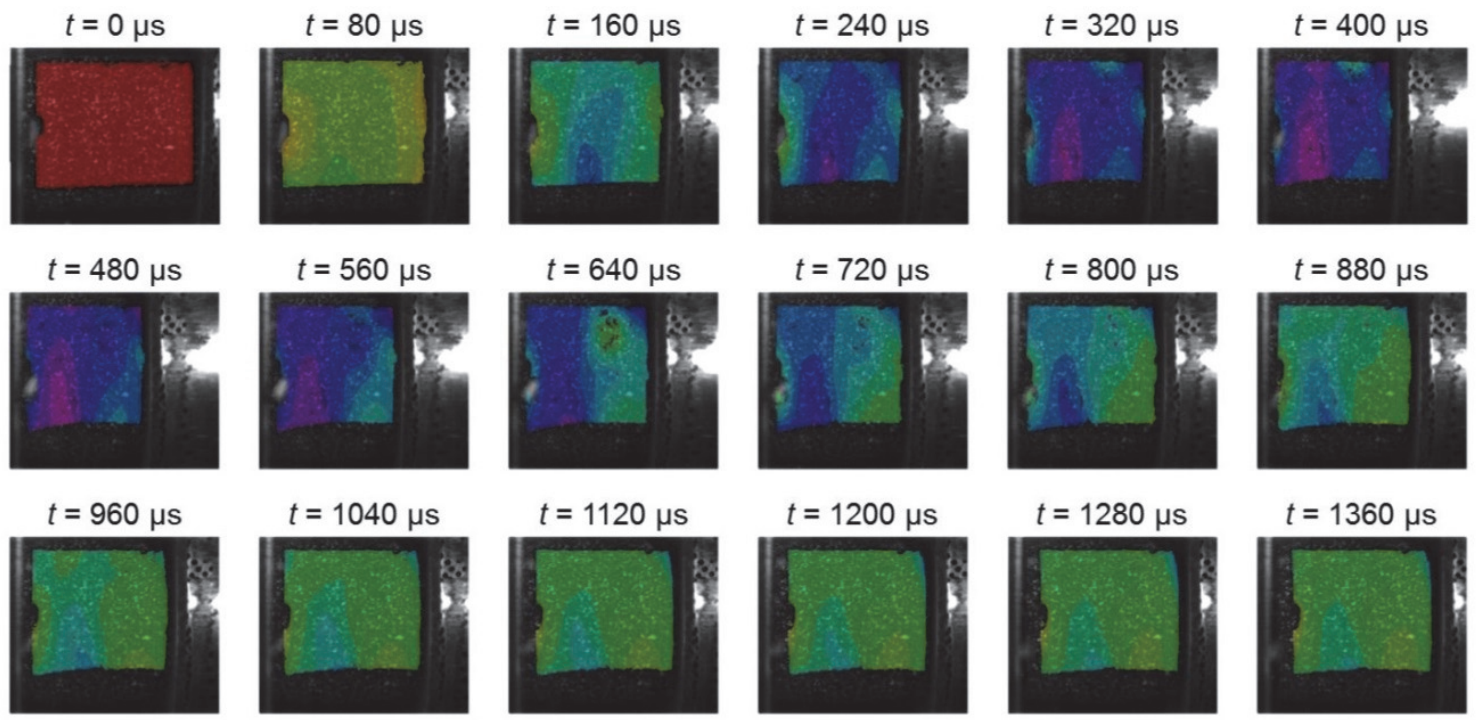

$$
t=1200 \mu \mathrm{s}
$$

$t=1280 \mu \mathrm{s}$
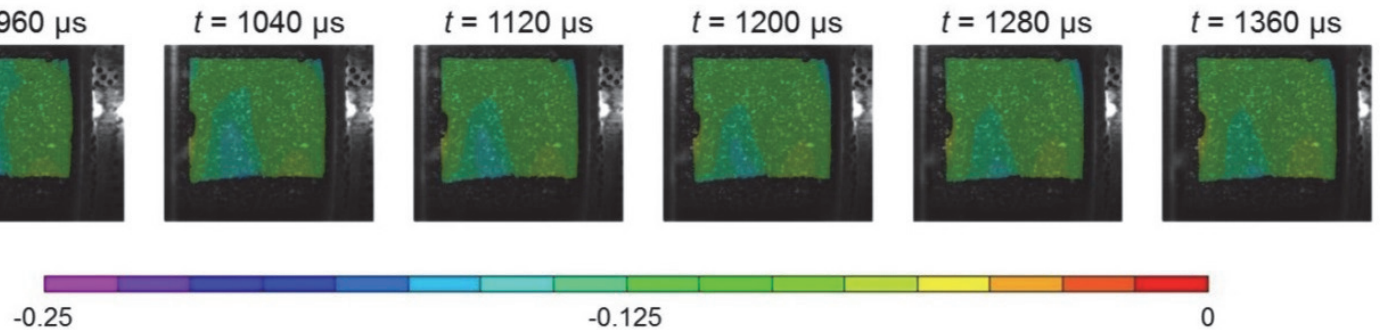

$-0.125$

Fig. 10- Contour maps showing the full-field distribution of axial strain at selected times after the impact. 

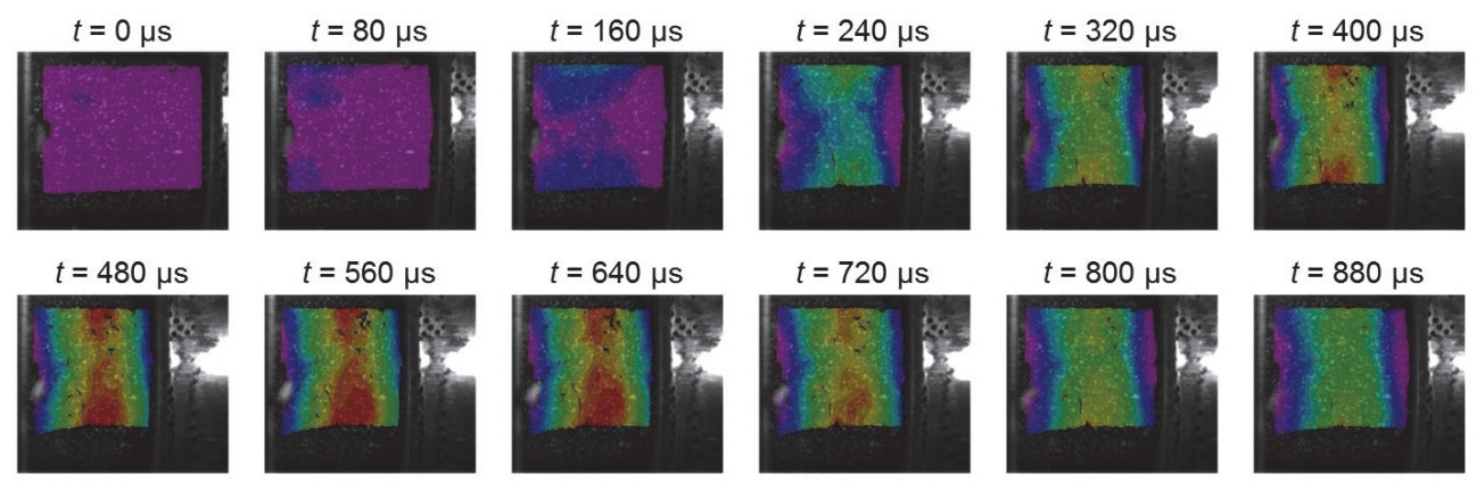

$t=880 \mu \mathrm{s}$

$$
t=960 \mu \mathrm{s}
$$

$$
t=1040 \mu \mathrm{s}
$$

$t=1120 \mu \mathrm{s}$

$t=1200 \mu \mathrm{s}$

$t=1280 \mu \mathrm{s}$
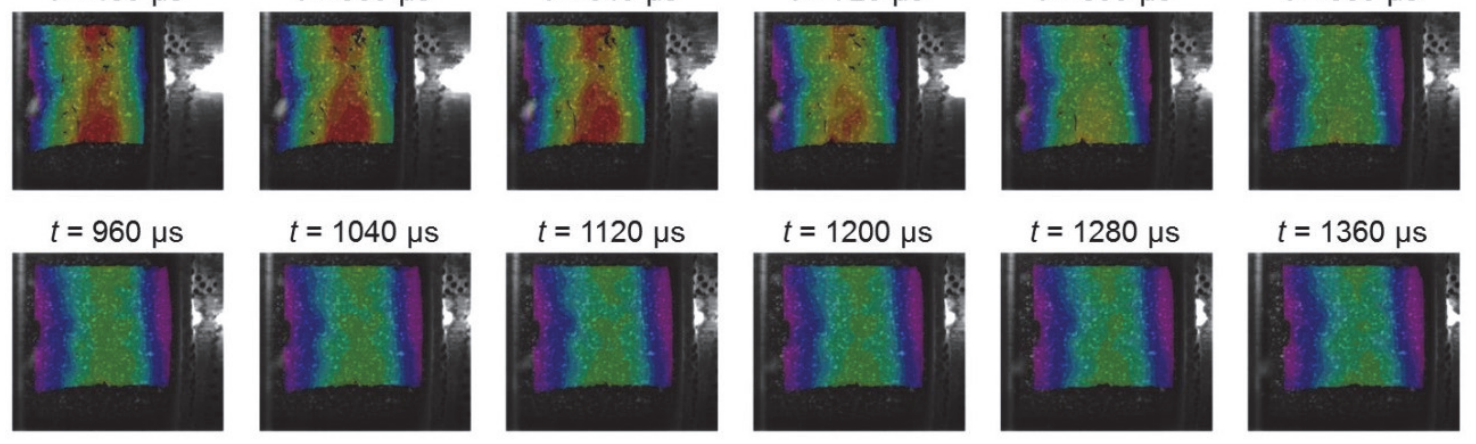

$t=1360 \mu \mathrm{s}$

0

0.0465

0.093

Fig. 11- Contour maps showing the full-field distribution of radial strain at selected times after the impact.

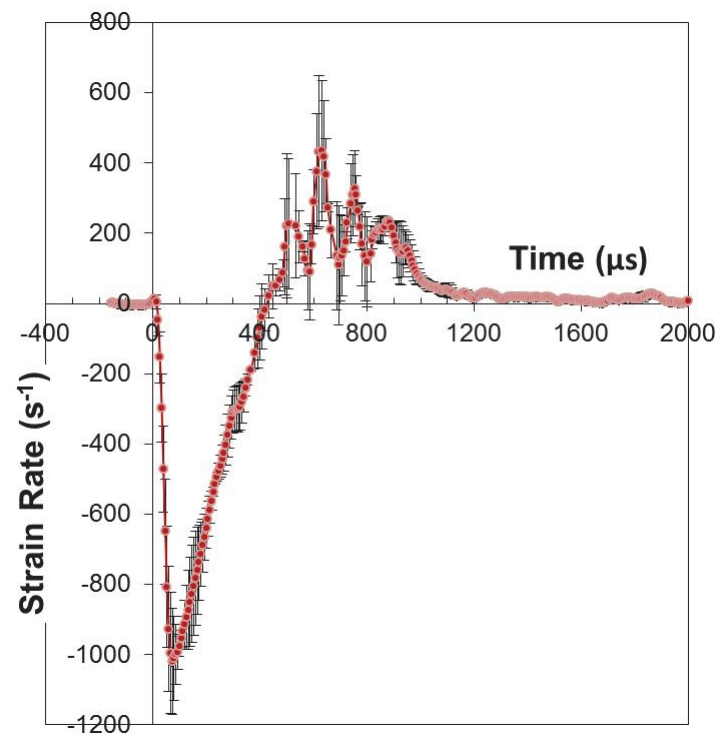

Fig. 12- Variation of strain rate in time. Error bars indicate the spatial variability of strain rate. 

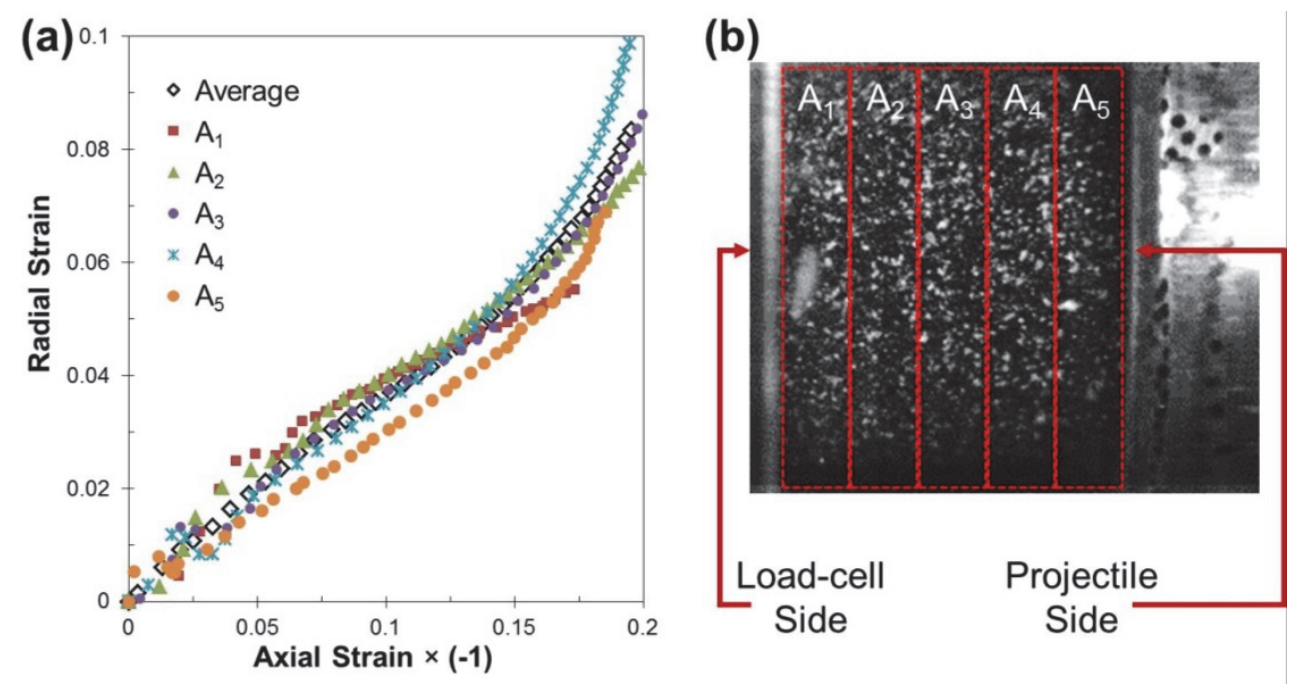

Fig. 13- (a) Variation of local radial strain with axial strain extracted from five representative sections shown in (b).

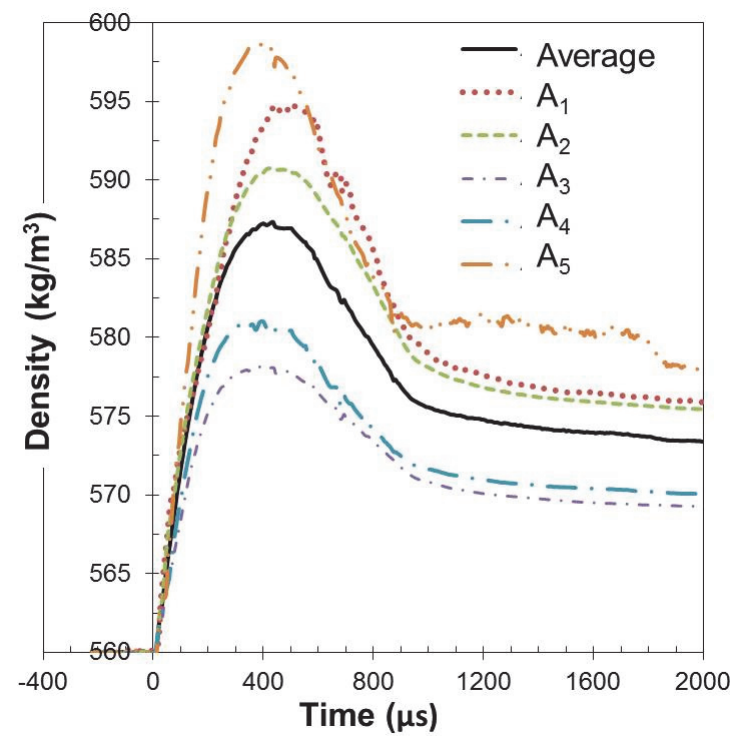

Fig. 14- Variation of local density in time for the representative sections shown in Fig. 13 b. 


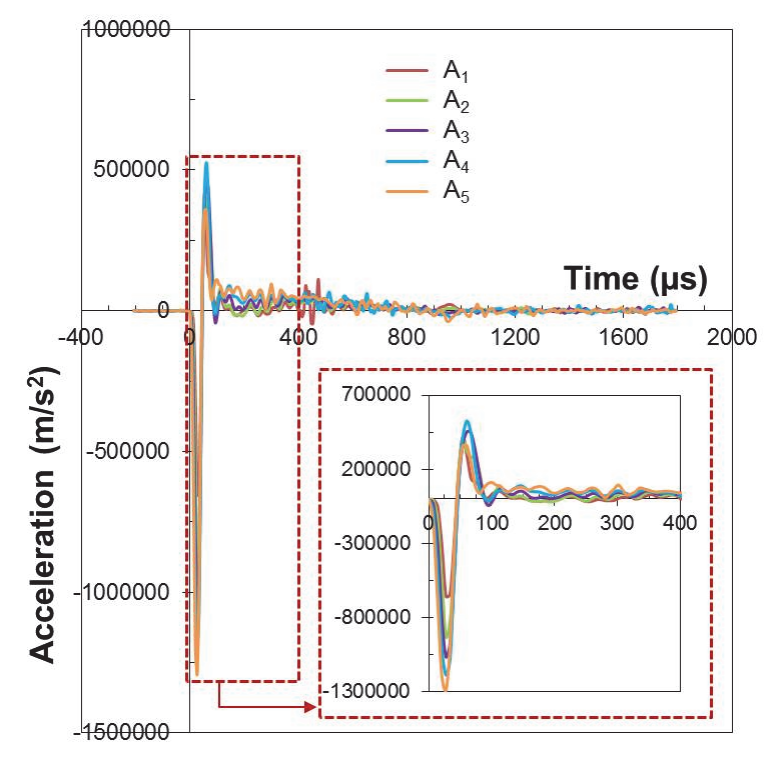

Fig. 15- Variation of local axial acceleration with time, extracted from the representative locations shown in Fig. 13b.

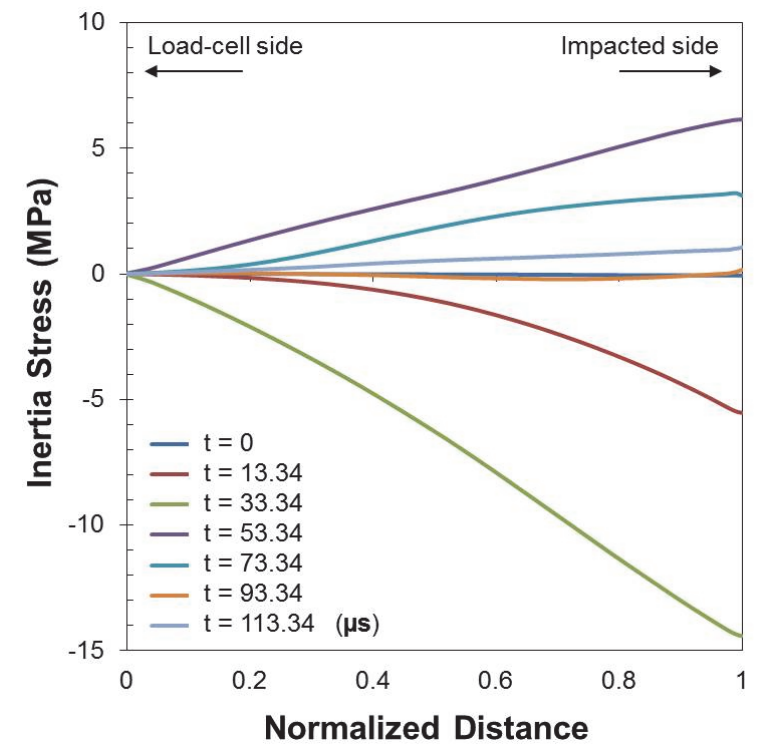

Fig. 16- Variation of inertia stress with respect to the normalized length of the specimen at different instants after impact, determined using full-field data. 


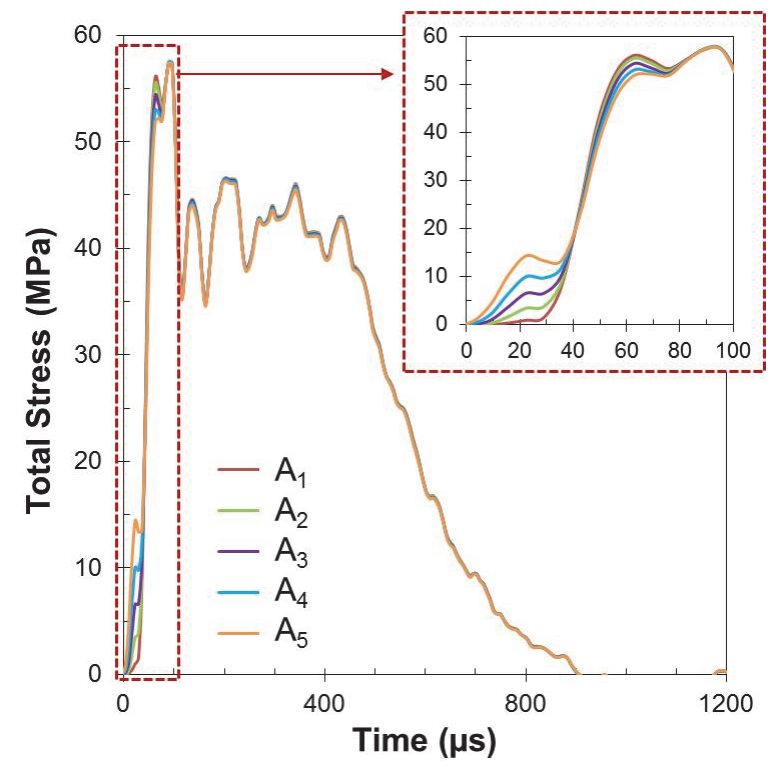

Fig. 17- Variation of total (inertia plus boundary measured) stress with time at different locations in specimen length.

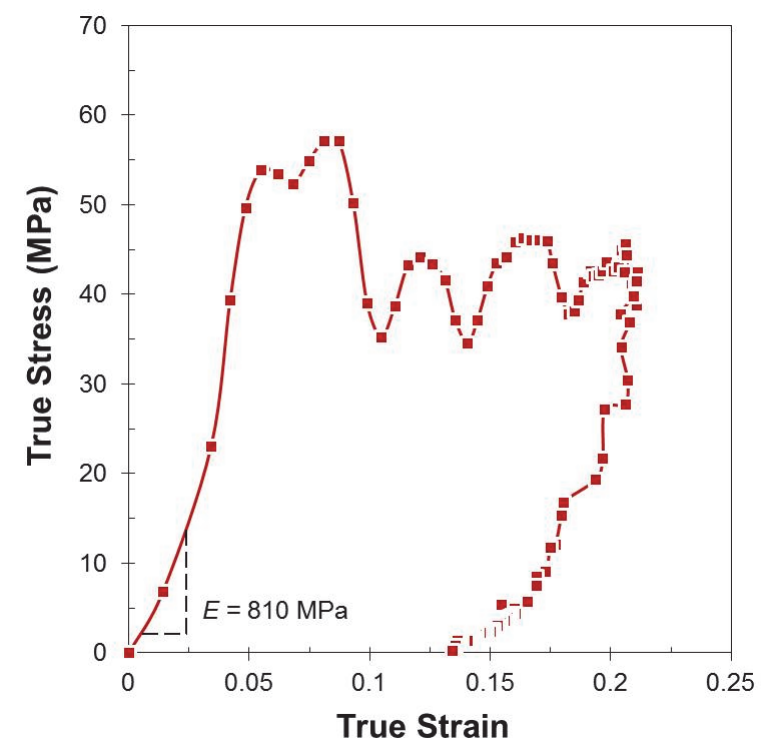

Fig. 18- True stress-strain curve during loading and unloading, calculated from full-field data. 


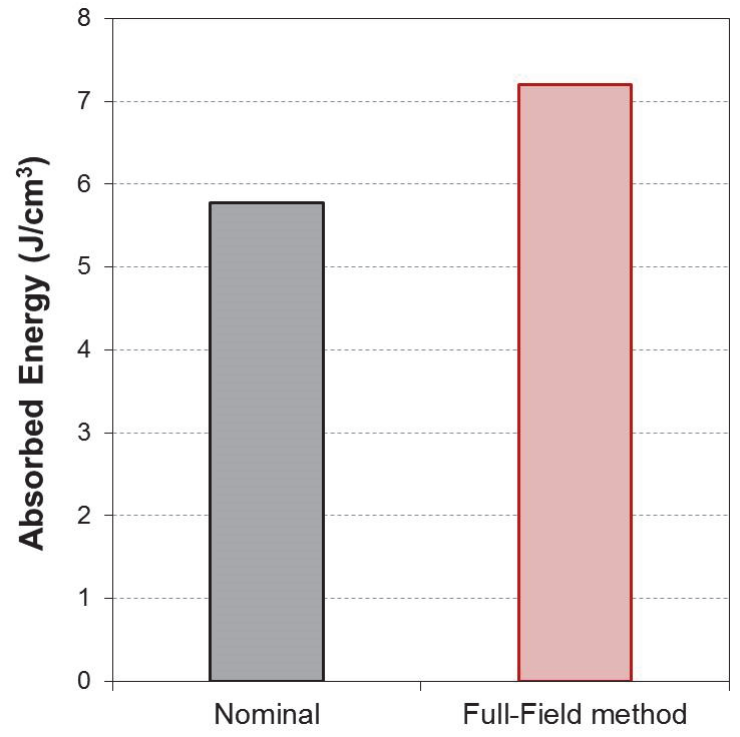

Fig. 19- Comparing specific energy values determined from two different methods. 